\title{
Cochliopodium minus, a scale-bearing amoeba isolated from organs of perch Perca fluviatilis
}

\author{
I. Dyková*, J. Lom, B. Macháčková \\ Institute of Parasitology, Academy of Sciences of the Czech Republic, CZ-370 05 Ceské Budèjovice, Czech Republic
}

\begin{abstract}
A scale-bearing amoeba isolated from gills and other organs of perch Perca fluviatilis Linnaeus, 1758 was identified as Cochliopodium minus Page, 1976. This assignment was suggested by light microscopy and confirmed by ultrastructural study of both the strain isolated from perch and the environmental strain of $C$. minus (CCAP 1537/1A) serving as a control. This has been the first electronmicroscopic definition of $C$. minus and, in addition, the first identification of an amoeba with a thecalike cover found to infect fish. The ability of the fish strain of $C$. minus to colonize gills and also internal body organs was proved experimentally.
\end{abstract}

KEY WORDS: Free living amoebae Cochliopodium minus - Ultrastructure - Systemic infection of fish

\section{INTRODUCTION}

The most important outbreaks of amoebic gill diseases (AGD) resulting in mortalities have been recorded in salmonids. Among the agents, Paramoeba spp. evidently play the most important role in marine cultures, while amoebae of the families Cochliopodidae and Thecamoebidae have been recorded as agents of severe gill lesions in freshwater farmed Oncorhynchus mykiss (Sawyer et al. 1974, 1975, Daoust \& Ferguson 1985, Noble et al. 1994, 1997). Unfortunately, none of the records of $A G D$ attributed to amoebae of the family Cochliopodidae included an exact diagnosis of the agent. Cochliopodium spp. have been recorded from a broad range of habitats, both marine and freshwater (Bark 1973, Page 1988) but most of the species were described at the light-microscopical level. Three species of the genus Cochliopodium isolated either from activated sludge of a sewage treatment plant or from a small sphagnum swamp were described in detail by Bark (1973). In addition, 1 strain of Cochliopodium isolated by Nagatani et al. (1981) from an aquarium sediment was used by Yamaoka et al. (1984) for a scale formation study. Structural components of scales were

•E-mail: iva@paru.cas.cz also examined in $C$. bilimbosum by Sadakane et al. (1996).

The goals of the present study were to report the isolation of a Cochliopodium sp. from the gills and other organs of perch Perca fluviatilis Linnaeus, 1758, and to present its ultrastructural features.

\section{MATERIALS AND METHODS}

The isolation, culture and cloning as well as maintenance of the cloned strain followed the general suggestions and systematic survey of culture methods of Kalinina \& Page (1992) and were also based on our experience from previous research on freeliving amoebae in freshwater fish (Dyková et al. 1996, 1997, 1998). The strain as well as the clone were cultured on non nutrient Bacto agar (Difco) seeded with Bacillus subtilis. In addition to lightmicroscopical methods of observation, transmission electron microscopy based on 2 fixation protocols, cacodylate buffered osmium tetroxide and glutaraldehyde with ruthenium red followed by postfixation with osmium tetroxide (Harris 1991), were applied to reveal surface architecture of the trophozoites. Amoebae cultured on agar plates were fixed in situ. Washed and pelleted material was embedded 
in Poly/Bed 812 - Araldite (Dykstra 1993). An identical procedure was applied to the strain of Cochliopodium minus Page, 1976 ordered from Culture Collection of Algae and Protozoa (CCAP 1537/1A) for comparison. The terminology used by Bark (1973), Nagatani et al. (1981) and Sadakane et al. (1996) was followed to allow comparison with previous descriptions of Cochliopodium strains. The ability of the isolated strain to colonize organs other than gills was tested experimentally in Heteropneustes fossilis, a bottom-dwelling fish species.

\section{RESULTS}

The Cochliopodium strain (accession No. 4694; a collection of strains is maintained at the Institute of Parasitology, Ceské Budějovice) was isolated from the liver, kidney, brain, spleen and gills of perch Perca fluviatilis caught in the Vltava river, South Bohemia, Czech Republic, in July 1991. Passage No. 18 of this strain was stored in liquid nitrogen. The clone was derived from passage No. 21 of this strain in March 1997.

\section{Light microscopy}

Locomotive and resting forms of trophozoites observed in hanging drops (Fig. 1) were round to oval in shape. The prominent, centrally located endoplasm was rich in granules and surrounded by a flat zone of hyaloplasm at the periphery. Pseudopodia of variable shape and length (maximum $6.5 \mu \mathrm{m}$ long) extended in all directions from the hyaloplasm.

When measured in a hanging drop of tap water, the average length $(L)$ of the most active trophozoites was $25.5(16-38) \mu \mathrm{m}$, the breadth $(B)$ was $26.5(12-52) \mu \mathrm{m}$ and the $L / B$ ratio was $1: 1.02$. The rate of locomotion averaged $54 \mu \mathrm{m} \mathrm{min}^{-1}$. The trophozoites were less active under the coverslip in distilled water, the rate of their locomotion being $17.4 \mu \mathrm{m} \mathrm{min} \mathrm{m}^{-1}$. Their $L$ was 21.4 $(16-28) \mu \mathrm{m}, B$ was $16.4(10-24) \mu \mathrm{m}$ and $L / B$ ratio was $1.3: 1$. The diameter of the nucleus averaged $3.4 \mu \mathrm{m}$ and of the nucleolus $2.3 \mu \mathrm{m}$. Trophozoites attached to coverslips of hanging drop preparations kept in a wet chamber for $12 \mathrm{~h}$ were observed to be inactive. They were almost discoid, having an $L$ of $26.0 \mu \mathrm{m}, B$ of $25.5 \mu \mathrm{m}$ and $L / B$ ratio $1.02: 1$.
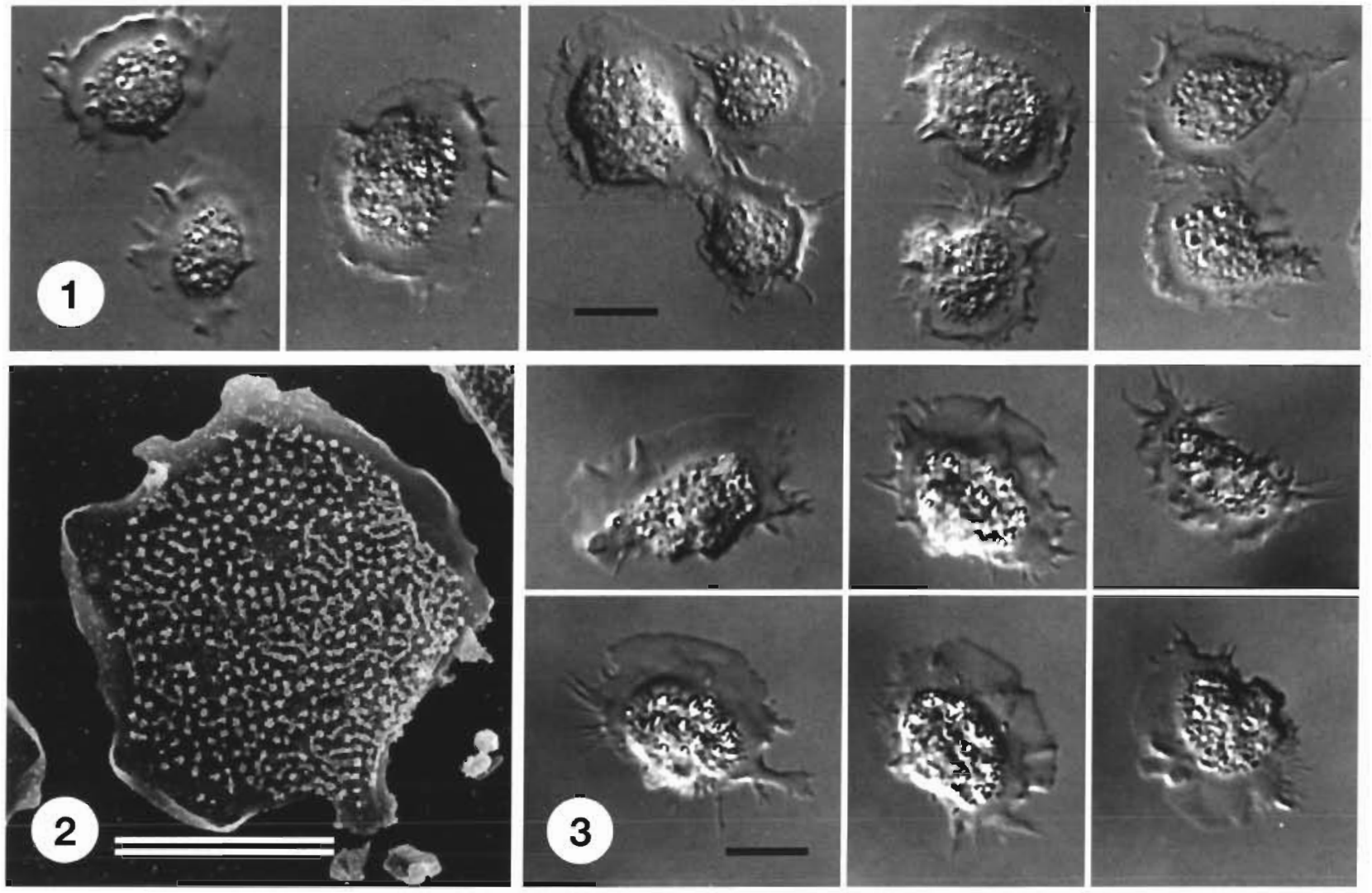

Figs. 1 to 3. Fig. 1. Trophozoites of Cochliopodium sp. (clone 4694/l isolated from organs of Perca fluviatilis) as observed in hanging drops. Nomarski DIC. Scale bar $=10 \mu \mathrm{m}$. Fig. 2. Cochliopodium sp. (clone 4694/I), scanning electron micrograph showing scale-bearing surface of the trophozoite. Scale bar $=10 \mu \mathrm{m}$. Fig. 3. Trophozoites of Cochliopodium minus (clone CCAP 1.537/1A; original designation ' 47 ' in Type Culture). Nomarski DIC. Scale bar $=10 \mu \mathrm{m}$ 
Light microscopy revealed the specific shape of the trophozoites. Concentric separation of the cytoplasm into the raised zone of granuloplasm and the flat rim of hyaloplasm was recorded. The presence of scales on the sur- face of trophozoites, which is of principal value for generic diagnosis, was at first only observed in transmission electron microscopy (see Figs. 4-8) and later also documented by scanning electron microscopy (Fig. 2).
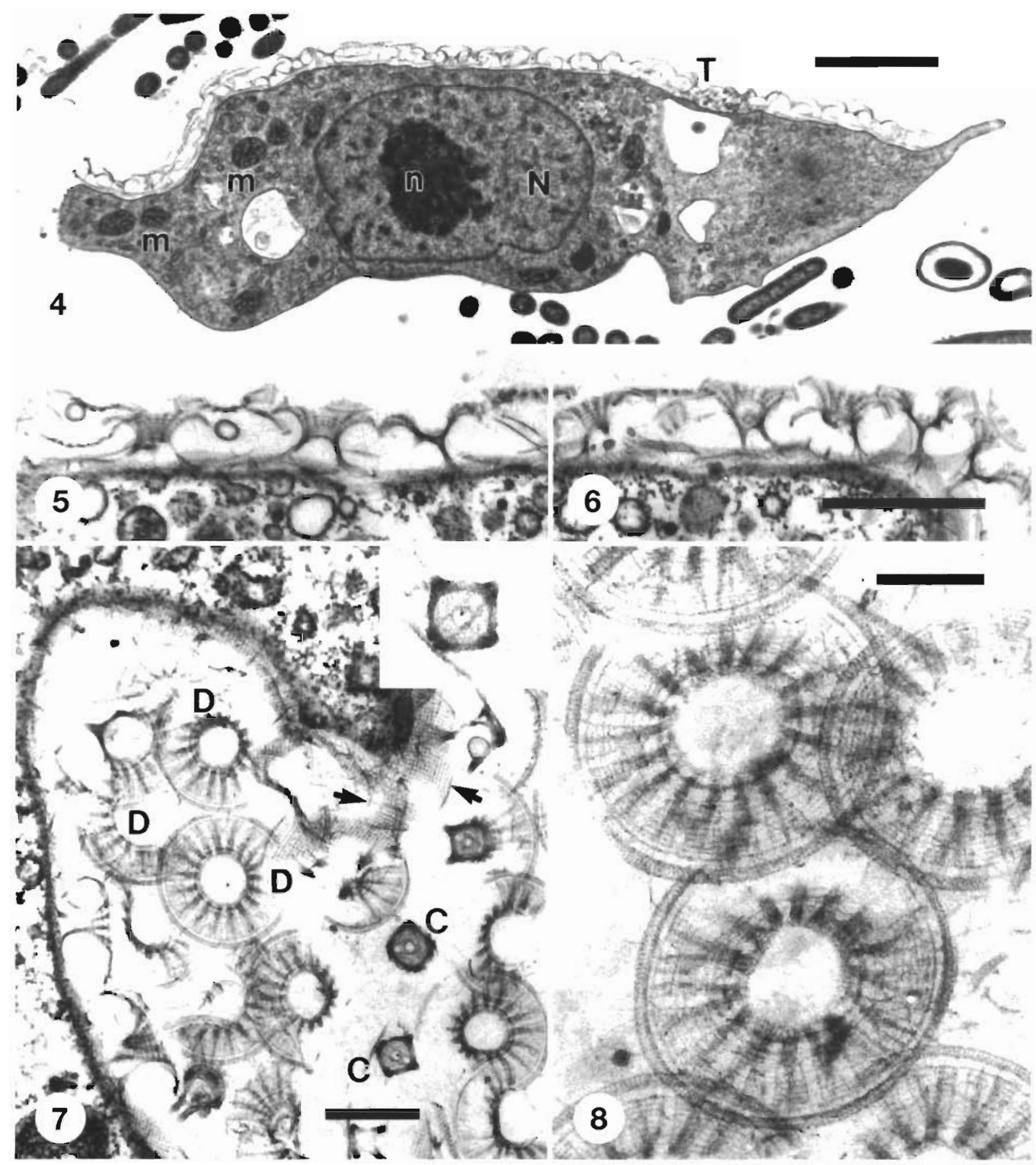

Figs. 4 to 8. Fig. 4. Trophozoite of Cochliopodium sp. (clone 4694/l) fixed on agar plate and sectioned in dorsoventral plane. Tectum $(\mathrm{T})$ consisting of scales, nucleus $(\mathrm{N})$, nucleolus $(\mathrm{n})$, mitochondria $(\mathrm{m})$ with tubular cristae. Scale bar $=2 \mu \mathrm{m}$. Figs. $5 \& 6$. Scale-bearing surface of trophozoites. Scale bar $=1 \mu \mathrm{m}$. Fig. 7. Microscales of Cochliopodium sp. (clone 4694/l) sectioned in various planes. Funnel-like part of scales in dorsal view (D). Rectangular transverse sections through the scale column (C). Fragments of scale base plates indicated by arrows. Scale bar $=500 \mathrm{~mm}$. Inset: Cross-section of the column. Fig. 8. Funnel-like part of scales in dorsal view showing web-like pattern with rays, concentric interconnections and striped rim. Scale bar $=200 \mathrm{~nm}$ 


\section{Transmission electron microscopy}

Cultured trophozoites fixed in situ were mostly elongated in cross section (Fig. 4). Their dorsal section was covered with a layer of slightly overlapping scales not extending to the peripheral hyaloplasm, while the ventral scale-free surface was attached to the agar The layer of scales extended up to $330 \mathrm{~nm}$ above the plasma membrane (Figs. $5 \& 6$ ). Nuclei were oval in shape and in some sections made the impression of having lobes. In the cytoplasm, symbiotic and phagocytized bacteria and granules of unknown origin were observed. Mitochondria contained branched tubular cristae and a prominent Golgi apparatus was observed several times close to the scale cover of the plasma membrane.

Each scale consisted of a flat lattice base plate attached to the plasma membrane of the trophozoite and a hollow column which at its capital flared open wide in the shape of a funnel (Figs. 5 \& 6). In the dorsal view (Fig. 8), the top, i.e. distal part, of the scale had the appearance of a spider web. The web-like structure consisted of 17 to 19 rays slightly widening towards the periphery which were concentrically interconnected (Figs. $7 \& 8$ ). The diameter of the funnel top was 560 to $620 \mathrm{~nm}$ and its perimeter was marked with a narrow striped rim (Fig. 8). The column of each scale was reinforced with 4 stalks which diverged toward the base plate (Figs. $5 \& 6$ ) and were shaped like birds' feet in the distal part. The transverse section through the middle part of the scale column was rectangular (Fig. 7), with sides equal in length to $180 \mathrm{~nm}$. When grown on agar plates, trophozoites formed double-walled cysts with diameters of 10 to $15 \mu \mathrm{m}$. The outer layer, i.e. the ectocyst, consisted of scales; the thin inner layer was closely apposed to the outer layer. In the outer scale layer of the cysts a scaleless aperture has always been observed.

\section{Taxonomic assignment}

The general morphology of the scale-bearing amoeba isolated from organs of Perca fluviatilis corresponded in all respects to the genus Cochliopodium (Hertwig \& Lesser, 1874) as redefined by Bark (1973). The assign-

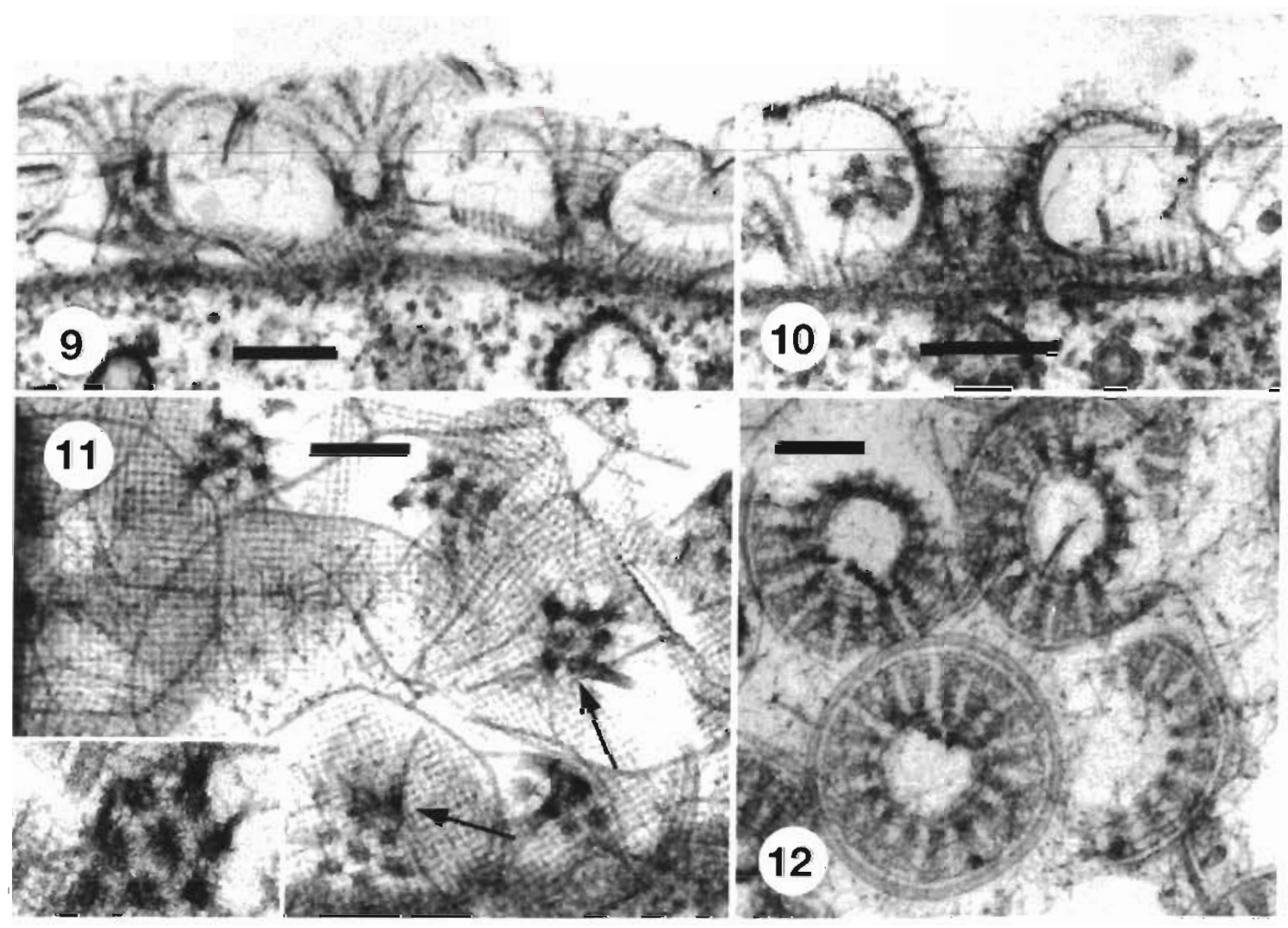

Figs. 9 to 12. Fig. 9. Cochliopodium minus (clone CAAP 1537/1A). Part of the tectum showing 3 scales sectioned in dorsoventral plane. Scale bar $=200 \mathrm{~nm}$. Fig. 10. Section through microscale of C. minus (CAAP 1537/lA) showing the top part of its column, which resembles the flared bell of a trumpet. Scale bar $=200 \mathrm{~nm}$. Fig. 11. Base plates of microscales with lattice structure and points of attachment of 4 stalks, which resemble bird's feet (arrows). Scale bar $=200 \mathrm{~nm}$. Inset: Detail of attachment. Fig. 12 . Scales of C. minus (clone CCAP $1537 / 1 \mathrm{~A}$ ) in dorsal view. Scale bar $=200 \mathrm{~nm}$ 
ment to the species $C$. minus Page, 1976 resulted from comparison of light microscopic and ultrastructural characteristics of the 2 clones: the newly isolated clone from perch (4694/1) and that from Culture Collection of Algae and Protozoa (CCAP 1537/1A) isolated by Page from a freshwater pond in 1965. Because data on the ultrastructure of C. minus (CCAP 1537/1A) were not available, we had to study this strain prior to comparison.

Trophozoites of Cochliopodium minus (CAAP 1537/ 1A) (Fig. 3) observed in hanging drops were identical and their measurements fitted in the size range of Cochliopodium sp. (4694/I). In addition, the type of scales, their shape and size (Figs. 9 to 12) corresponded to those of the Cochliopodium sp. under study. The heights of scales above the plasma membrane, the diameters of basal plates and the diameters of the distal parts of scales differed only insignificantly (on the scale of nanometers). The same applied to the number of rays in distal part of scales (16 or 17 in C. minus from CCAP, and often more, (17 to 19 , in C. minus from Perca fluviatilis)

\section{Experimental infection}

Cochliopodium minus was isolated from gills and other organs (kidney, liver and brain) of 3 out of 6 specimens of Heteropneustes fossilis, a bottom-dwelling fish species, kept for $5 \mathrm{wk}$ in water enriched with trophozoites and cysts from culture of $C$. minus (4964/1). C. minus was not isolated from any of 5 control fishes of the same origin.

\section{DISCUSSION}

Comparison of the size of trophozoites and scale ultrastructure of the clone isolated from perch with the relevant data available from 4 species of the genus (1 named and 3 unnamed species) excluded 2 Cochliopodium species: the type species $C$. bilimbosum Auerbach, 1856 and the species designated as Cochliopodium sp. 3 by Bark (1973). Features similar to the present Cochliopodium sp. were described in Cochliopodium sp. 2 by Bark (1973) and by Nagatani et al. (1981) in strain NYS, which Yamaoka et al. (1984) used (under the name Cochliopodium sp.) for scale formation study. While the sizes (mean $L$ and $B$ ) of trophozoites, diameters of nuclei and nucleoli and the size of scales fitted in the same range for the Cochliopodium $\mathrm{sp}$. under study and Cochliopodium sp. 2 described by Bark (1973), the scale morphology differed. In Cochliopodium sp. 2 the 4-ribbed column was topped with a flat wheel-shaped structure, which in the dorsal view had 2 concentric rings and 20 spokes. The ultrastructural comparison of scales in the present Cochliopodium sp. and Cochliopodium sp. strain NYS revealed similarity in the shape but divergence in size. All scale metric data (the height above the plasma membrane, the diameter of the top of the funnel and the number of rays in the web-like structure) were larger in the NYS strain. The same applied to the diameter of trophozoites measured by light microscopy

In addition to the type species Cochliopodium bilimbosum Auerbach, 1856, 13 species of the genus Cochliopodium named from 1856 to 1926 were listed by Bark (1973). Since only light-microscopical features were available in the species described before, Bark (1973) faced an extremely difficult task trying to compare his own findings with the previously described species. Thanks to his study, the light-microscopical description of the type species, C. bilimbosum, was supplemented by ultrastructural data and the specific scale structure was recognized. Thus, only 1 of the 3 microscale patterns discovered by Bark (1973) could be attributed to the previously described Cochliopodium species. The other 2 types of scales characterize the 2 still unnamed different Cochliopodium spp. In addition to the type species, Page (1988) included in the genus Cochliopodium 2 other species, C. actinophorum (Auerbach, 1856) and C. minus Page, 1976. While $C$. actinophorum and $C$. bilimbosum, the species with large trophozoites and light-microscopically perceptible microscales, differ essentially, Cochliopodium sp. isolated from organs of perch could be identified with C. minus (CAAP 1537/1A) isolated by Page from a freshwater pond (Madison, Wisconsin, USA).

In older classifications (Levine et al. 1980) the genus Cochliopodium was assigned to the order Arcellinida Kent, 1880, i.e. among testate amoebae. Page (1987), however, established a special order, Himatismenida Page, 1987, for this genus, which he regarded as a transitional category between Gymnamoebia Haeckel 1862 and Testacealobosia de Saedeleer, 1934, Irrespective of their actual taxonomic position, this is the first amoeba with a test-like cover (tectum) of unattached cell surface recovered as a systemic parasite of fish.

Acknowledgements. This work was funded by the Grant Agency of the Academy of Sciences of the Czech Republic (Research project No. A6022802). The skillful technical assistance of Mr Jaroslav Bica was of great help

\section{LITERATURE CITED}

Bark AW (1973) A study of the genus Cochliopodium Hertwig and Lesser 1874. Protistologica 9:119-138

Daoust PY, Ferguson HW (1985) Nodular gill disease in rainbow trout, Salmo gairdneri Richardson. J Fish Dis 8 $511-522$ 
Dyková I, Lom J, Macháčková B, Sawyer I (1996) Amoebic infections in goldfishes and granulomatous lesions. Folia Parasitol 43:81-90

Dyková L, Macháčková B, Pecková H (1997) Amoebae isolated from organs of farmed tilapias Oreochromis niloticus. Folia Parasitol 44:81-90

Dyková I, Lom J, Macháčková B, Pecková H (1998) Vexillifera expectata sp. n. and other non-encysting amoebae isolated from organs of freshwater fish. Folia Parasitol 45: $17-26$

Dykstra MJ (1993) A manual of applied techniques for biological electron microscopy. Plenum Press, New York, p 49

Harris R (1991) Electron microscopy in biology. Oxford University Press, Oxford, p 8

Kalinina LV, Page FC (1992) Culture and preservation of naked amoebae. Acta Protozool 31:115-126

Levine ND, Corliss JO, Cox FEG, Deroux G, Grain J, Honigberg BM, Leedale GF, Loeblich AR, Lom J, Lynn D, Merinfeld EG, Page FC, Poljansky G, Sprague V, Vavra J, Wallace FG (1980) A newly revised classification of the Protozoa. J Protozool 27:37-58

Nagatani Y, Yamaoka I, Sato N (1981) Scale structure of the external surface of an amoeba. Zool Mag 90:112-115

Noble A, Herman R, Noga E, Bullock G (1994) Description of a recurrent amoeba gill infestation in rainbow trout (Oncorhynchus mykiss) cultured in a semiclosed recircu-

Editorial responsibility: Wolfgang Körting,

Hannover, Germany lating system. Int Symp Aquat Anim Health, Seattle, Washington, September 4-8, 1994, Abstract W-10.1

Noble AC, Herman RL, Noga EJ, Bullock GL (1997) Recurrent amoebic gill infestation in rainbow trout cultured in a semiclosed water recirculation system. I Aquat Anim Health 9:64-69

Page FC (1987) The classification of 'naked' amoebae (Phylum Rhizopoda). Arch Protistenkd 133:199-217

Page FC (1988) A new key to freshwater and soil gymnamoebae with instructions for culture. Freshwater Biological Association, Ambleside

Sadakane K, Murakami R, Yamaoka I (1996) Cytochemical and ultrastructural studies of the scales of the amoeba Cochliopodium bilimbosum (Testacea). Eur J Protistol 32: 311-315

Sawyer TK, Hnath JG, Conrad JF (1974) Thecamoeba hoffmani sp. n. (Amoebida: Thecamoebidaae) from gills of fingerling salmonid fish. J Parasitol 60:677-682

Sawyer TK, Hoffman GL, Hnath JG, Conrad JF (1975) Infection of salmonid fish gills by aquatic amoebas (Amoebida: Thecamoebidae). In: Ribelin WE, Migaki G (eds) The pathology of fishes. The University of Wisconsin Press, Madison, p 143-150

Yamaoka I, Kawamura N, Mizuno M, Nagatani Y (1984) Scale formation in an amoeba Cochliopodium sp. J Protozool $31: 267-272$

Submitted: July 6, 1998; Accepted: September 1, 1998 Proofs received from author(s): November 26, 1998 